\title{
Harmonic behavior of metallic glasses up to the metastable melt
}

\author{
A. Meyer \\ Physik-Department, Technische Universität München, 85747 Garching, Germany \\ and European Synchrotron Radiation Facility, 38043 Grenoble, France \\ J. Wuttke and W. Petry \\ Physik-Department, Technische Universität München, 85747 Garching, Germany
}

A. Peker

Keck Laboratory of Engineering Materials, California Institute of Technology, Pasadena, California 91125

R. Bormann

Institut für Werkstofforschung, GKSS, 21502 Geesthacht, Germany

G. Coddens

Laboratoire Léon Brillouin, CEN-Saclay, 91191 Gif-sur-Yvette, France

L. Kranich

Physik-Department, Technische Universität München, 85747 Garching, Germany

O. G. Randl and H. Schober*

Institut Laue-Langevin, 38042 Grenoble, France

(Received 3 November 1995)

In two amorphous alloys $\mathrm{ZrTiCuNiBe}$ and $\mathrm{ZrAlNiCu}$ coherent neutron scattering has been measured over five decades in energy, including measurements in the metastable melt of a metallic alloy more than $80 \mathrm{~K}$ above $T_{g}$. In the vibrational spectra a pronounced "boson" peak is found: Even in crystallized samples the density of states exceeds the Debye $\omega^{2}$ model, and in the amorphous state low-frequency vibrations are further enhanced. The peak position shows no dispersion in $q$, while intensities are strongly correlated with the static structure factor. Over the full energy range the temperature dependence is strictly harmonic. From high-energy resolution measurements we establish lower bounds for the temperatures at which structural $\alpha$ and fast $\beta$ relaxation become observable. [S0163-1829(96)01218-0]

\section{INTRODUCTION}

The low-energy vibrational spectra of many glasses deviate in a characteristic way from Debye's plane-wave density of states, the excess modes being designated as the "boson peak." On heating towards and above the glass transition, a sequence of additional relaxational modes sets in. Very similar behavior is observed in chemically quite dissimilar samples, ${ }^{1}$ and there is an ongoing discussion about possible interrelations between the strength of the chemical bonds, the width of the first diffraction peak, the spectral broadening of structural relaxation, and the temperature dependence thereof. $^{2-4}$

So far it is not known how metallic glasses accomodate within these schemes. Conventional alloys inevitably crystallize on approaching the glass transition. Only recently ${ }^{5,6}$ four- and five-component glasses have been developed that open the possibility of performing day long experiments in the metastable melt far above the calorimetric glass transition. By means of broad band inelastic neutron scattering we have studied the vibrational properties and searched for relaxational motion in two different metallic glasses.

\section{EXPERIMENTAL}

The formula $\mathrm{Zr}_{65} \mathrm{Al}_{7.5} \mathrm{Ni}_{10} \mathrm{Cu}_{17.5}$ was taken from the literature; ${ }^{5}$ the other alloy's composition $\mathrm{Zr}_{46.8} \mathrm{Ti}_{8.2} \mathrm{Cu}_{7.5} \mathrm{Ni}_{10} \mathrm{Be}_{27.5}$ was improved with respect to pre-

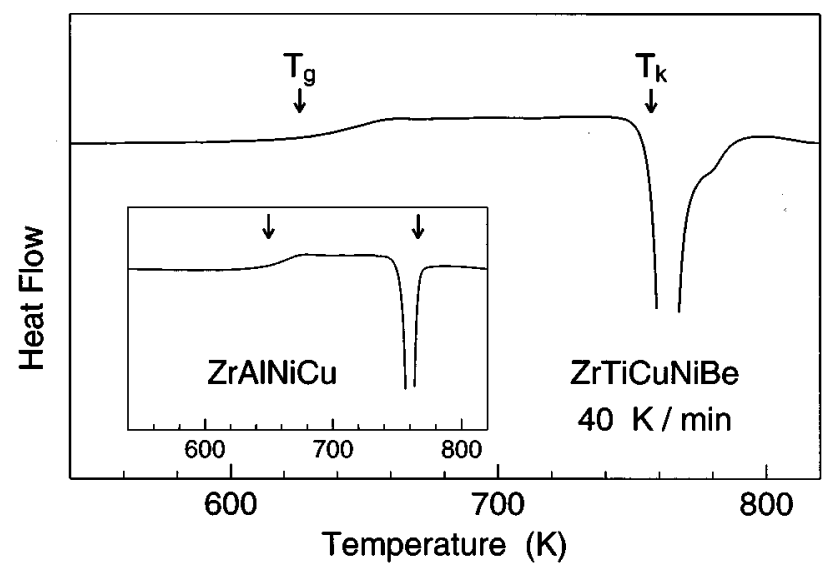

FIG. 1. DSC scans of the glassy alloys with a heating rate of $40 \mathrm{~K} / \mathrm{min}$ using a Netzsch STA 409 scanning calorimeter. 
TABLE I. Calorimetric glass transition $T_{g}$ and crystallization temperature $T_{k}$ vs heating rate.

\begin{tabular}{lcccc}
\hline \hline & \multicolumn{2}{c}{ ZrTiCuNiBe } & \multicolumn{2}{c}{ ZrAlNiCu } \\
& $T_{g}$ & $T_{k}$ & $T_{g}$ & $T_{k}$ \\
& $(\mathrm{~K})$ & $(\mathrm{K})$ & $(\mathrm{K})$ & $(\mathrm{K})$ \\
\hline $40 \mathrm{~K} / \mathrm{min}$ & 626 & 757 & 643 & 757 \\
$20 \mathrm{~K} / \mathrm{min}$ & 613 & 741 & 643 & 739 \\
$2 \mathrm{~K} / \mathrm{min}$ & 596 & 706 & 624 & 700 \\
Quasi-stationary & 582 & & 605 & \\
\hline
\end{tabular}

vious publications ${ }^{6}$ and has an even wider range of stability in the melt. More than $75 \mathrm{~m}$ of $\mathrm{ZrAlNiCu}$ ribbons with a section of about $2.5 \times 0.06 \mathrm{~mm}^{2}$ were prepared by conventional melt spinning. For preparing amorphous $\mathrm{ZrTiCuNiBe}$ relatively low cooling rates are required so that bulk material could be obtained by casting the melt into copper molds and by suiting the ingot to $35 \times 5 \times 1.1 \mathrm{~mm}^{3}$. The sample material was characterized by $\mathrm{x}$-ray diffraction and thermal analysis. The $\mathrm{x}$-ray structure factor behaved as expected for an amorphous solid and showed no traces of crystallization. By differential scanning calorimetry (DSC) we found that the samples can be heated more than $100 \mathrm{~K}$ above $T_{g}$ before crystallization sets in (Fig. 1). Both the glass transition $T_{g}$ and the crystallization temperature $T_{k}$ depend of course on the heating rate. Table I gives a summary of thermal properties, including an estimation for the quasistationary situation of neutron-scattering experiments (scans of about $5 \mathrm{~h}$ duration at a given temperature).

Preliminary experiments had shown that surface oxidation is a main cause for precocious crystallization. The samples were therefore sealed under $\mathrm{He}$ atmosphere in $\mathrm{Al}$ containers. For neutron scattering a hollow cylinder geometry (height $52 \mathrm{~mm}$, diameter $22 \mathrm{~mm}$, thickness $1.1 \mathrm{~mm}$ ) was chosen. Three samples of each composition were prepared, all of them being deliberately crystallized at the end of the measuring campaign. To determine the melting point of the alloys, DSC scans were performed with samples sealed under $\mathrm{He}$ atmosphere in Gold pans. For $\mathrm{ZrTiCuNiBe}$ the melting point is $908 \mathrm{~K}$, while for $\mathrm{ZrAlNiCu} T_{m}=1098 \mathrm{~K}$ is much higher. Comparing the interval $T_{m}-T_{g}$ with the stability range $T_{k}-T_{g}$ of the undercooled melt, we anticipate that $\mathrm{ZrTiCuNiBe}$ is the more promising candidate for the search of relaxational processes.

In this work, we have combined four setups on three spectrometers to cover energy transfers from less than $1 \mu \mathrm{eV}$ up to at least $100 \mathrm{meV}$ : the thermal neutron time-offlight machine DN6 of the Kernforschungszentrum Karlsruhe at the Centre d'Études Nucléaires de Grenoble (wavelength $\lambda=2.2 \AA$, resolution full width at half maximum $\delta E=950 \mu \mathrm{eV}$, wave number range at zero energy transfer $q=1.2-4.3 \AA^{-1}$ ); the cold neutron time-offlight spectrometer Mibémol at the LLB, Saclay, with $\lambda=5 \AA$ for maximum flux $\left(\delta E=140 \mu \mathrm{eV}, 0.8-2.4 \AA^{-1}\right)$ and in another setup with $\lambda=8.5 \AA$ for higher resolution $\left(\delta E=30 \mu \mathrm{eV}, q=0.5-1.4 \AA^{-1}\right)$; and the backscattering instrument IN10 at the ILL, Grenoble $[\lambda=3.27 \AA$, $\delta E=4.2 \mu \mathrm{eV}, q=1.7-3.7 \AA^{-1}$ (Ref. 7)].

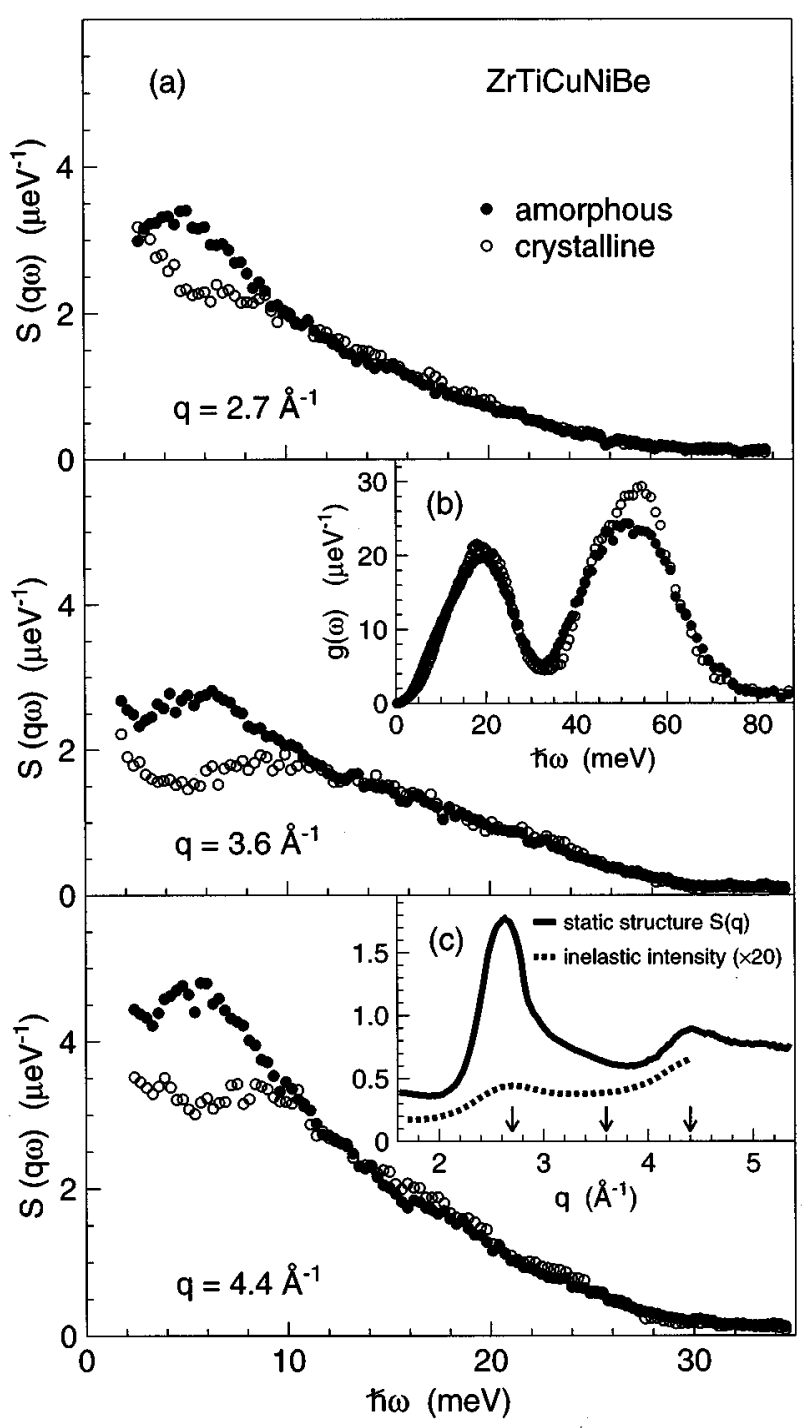

FIG. 2. Vibrational spectra of ZrTiCuNiBe before and after crystallization, measured at room temperature on DN6. (a) Scattering law $S(q \omega)$ for different $q$. (b) Density of vibrational states $g(\omega)$ evaluated in incoherent approximation. The total number of states was normalized to 1 . (c) Static structure factor $S(q)$ compared to the inelastic intensity integrated over the interval 2.4-10 meV. The arrows in (c) indicate the wave numbers chosen in (a).

\section{VIBRATIONAL PROPERTIES}

Vibrational modes are studied by time-of-flight spectroscopy in the meV range. In some simple experimental situations incoherent neutron scattering yields a fiducial density of vibrational states (DOS) $g(\omega)$. However, our samples contain four or five different elements with predominantly (about 90\%) coherent scattering so that a DOS can be determined only in a very first approximation. Treating $S(2 \theta, \omega)$ as if it were an incoherent scattering law, we obtain for every detector angle $2 \theta$ another $g(\omega)$. By averaging over the full accessible $q$ range $\left(2 \theta=23^{\circ} \ldots 99^{\circ}\right)$ (incoherent approximation $\left.^{8}\right)$ we obtain a $g(\omega)$ which may give at least a rough idea of the spectral distribution of vibrational modes [Figs. 2(b), and 3(b)]. 


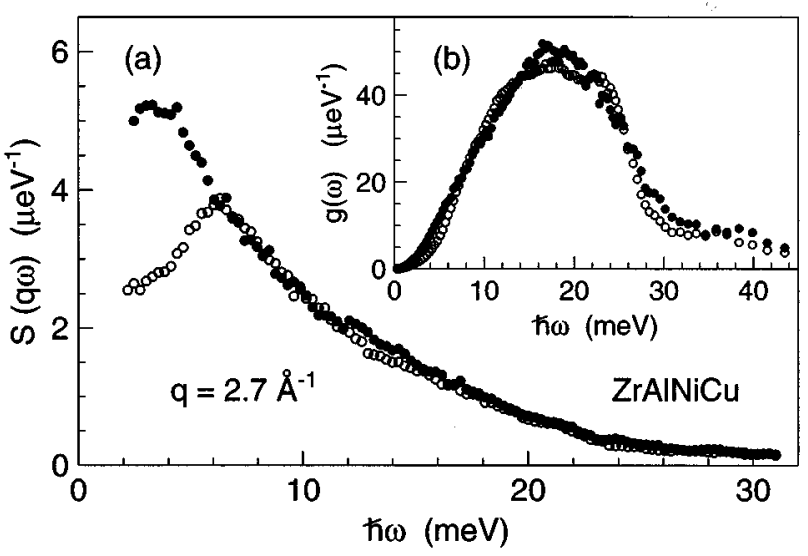

FIG. 3. Vibrational spectra of $\mathrm{ZrAlNiCu}$ before and after crystallization, measured and plotted as in Figs. 2(a) and 2(b).

Up to about $30 \mathrm{meV}$ both alloys show similar behavior, with a broad maximum around $20 \mathrm{meV}$. A second peak around $50 \mathrm{meV}$ that occurs only in ZrTiCuNiBe may be ascribed to vibrations of the light Be atoms. Above $10 \mathrm{meV}$ the spectra of amorphous and crystallized samples nearly coincide: the eigenfrequencies of high-lying modes in which near neighbors oscillate against each other appear rather insensitive to the presence or absence of long-range crystalline order.

Below $10 \mathrm{meV}$ vibrational modes are studied better in terms of $S(q \omega)$ which in one-phonon approximation is proportional to $g(\omega) / \omega^{2}$. Assuming a Debye DOS, a constant $S(q \omega)$ should be expected. Instead, as most other amorphous substances, our metallic glasses show a "boson" maximum of $S(q \omega)$ [Figs. 2(a) and 3(a)] that reveals an excess of lowenergy modes with respect to a Debye solid. In ZrTiCuNiBe the boson peak is located at about $5 \mathrm{meV}$ and shows no dispersion in $q$; the intensity modulates weakly in phase with

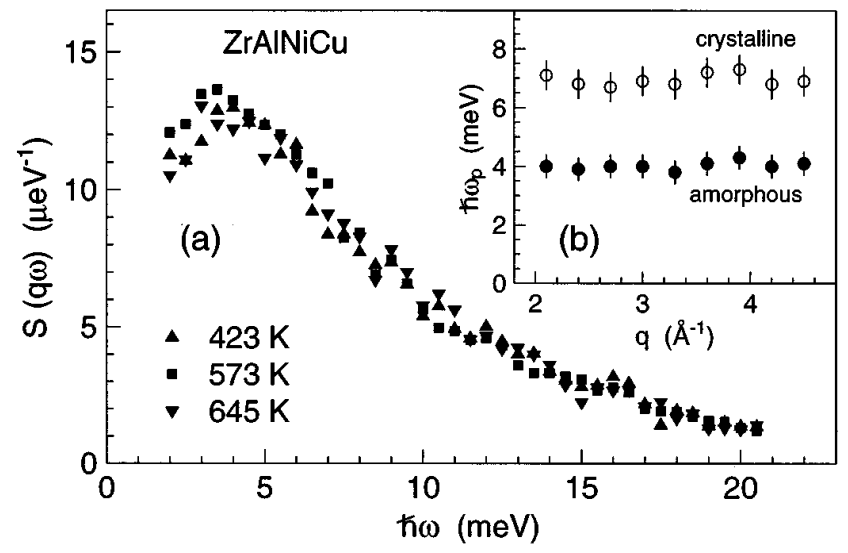

FIG. 4. (a) Vibrational spectra of $\mathrm{ZrAlNiCu}$ in the amorphous state, measured on Mibémol at $q=2.6 \AA^{-1}$, at different temperatures up to the metastable melt, rescaled with the Bose occupancy $n(\omega, 573 \mathrm{~K}) / n(\omega, T)$ in order to demonstrate harmonic behavior over the full frequency range. (b) Frequency $\omega_{p}$ of the inelastic maximum of $S(q \omega)$ as function of $q$. Within experimental error, no dispersion could be detected.
$S(q)$ [Fig. 2(c)]. ZrAlNiCu behaves similarly.

In this context one should note that a peak in $S(q \omega)$ is observed not only in the amorphous samples but also (at somewhat higher frequencies) in their crystalline counterparts (at $6 \mathrm{meV}$ in $\mathrm{ZrAlNiCu}$, and less pronounced at $9 \mathrm{meV}$ in $\mathrm{ZrTiCuNiBe}$ ). Above this peak which shows also no dispersion in $q$ [Fig. 4(b)] amorphous and crystalline spectra merge as discussed before: above a certain frequency, vibration modes do not change much when a disordered system crystallizes into a complicated unit cell.

Up to here, our observations resemble those made in conventional, binary glasses like $\mathrm{Cu}_{46} \mathrm{Zr}_{54}$ (Ref. 9) and $\mathrm{Mg}_{70} \mathrm{Zn}_{30},{ }^{10,11}$ where, however, the boson peak either showed dispersion in $q$ (Ref. 11) or could not be fully resolved. ${ }^{9}$ In these studies, an inelastic peak in the crystalline phase's $S(q, \omega)$ has been ascribed to the onset of dispersion near the lowest zone boundary. ${ }^{11,12}$ This interpretation seems to be in conflict with the absence of $q$ dispersion in our measurements. On the other hand, one might argue that boundaries of pseudo-Brillouin zones are considerably smeared out in complicated structures.

Investigations of vibrational spectra in conventional glasses have most often been performed at room temperature. In the context of our search for relaxational modes (Sec. IV) we extended our measurements over a wider temperature range. The thermal stability of our four- and five-component samples allowed vibrational spectroscopy above $T_{g}$. Over the full temperature range, scattering intensities grew as expected from Bose-Einstein statistics. In Fig. 4, rescaled spectra $S(q \omega) / n(\omega, T)$ are shown to coincide within the experimental noise level of about $5 \%$. Even more precise evidence for strictly harmonic behavior over a wide temperature range reaching far into the metastable melt was obtained from elastic measurements as described in the next section.

The vibrational spectra of amorphous materials are often decomposed into a Debye component plus an excess contribution that is ascribed to "glass-typical" modes, fitted by some model function, and possibly brought into connection with the onset of relaxational modes at higher temperatures. ${ }^{13}$ The presence of non-Debye components in the polycrystalline spectra and the strictly harmonic temperature dependence of all excitations recommend more caution: the vibrational spectra may eventually turn out to be less universal and less strongly correlated with the glass transition dynamics than used to be thought. ${ }^{14}$ Our observations rather support a picture in which the boson peak is ascribed to localized vibrations that do not show more anharmonicity than any other phonon mode.

\section{SEARCH FOR RELAXATIONAL MOTION}

With high-energy resolution measurements we have searched for the onset of relaxational motion. Structural $(\alpha)$ relaxation should become observable as quasielastic broadening on IN10. In previous studies on organic liquids, the linewidth of structural relaxation was found to increase strongly with $q ;{ }^{15}$ therefore the new configuration of IN10 with a maximum $q=3.7 \AA^{-1}$ was particularly valuable. A broadening of $1 / 10$ of the resolution width would easily have been detected in $S(q \omega)$. Nevertheless, up to $653 \mathrm{~K}$ in ZrTi$\mathrm{CuNiBe}$ no quasielastic broadening was observed [Fig. 5(a)]. 

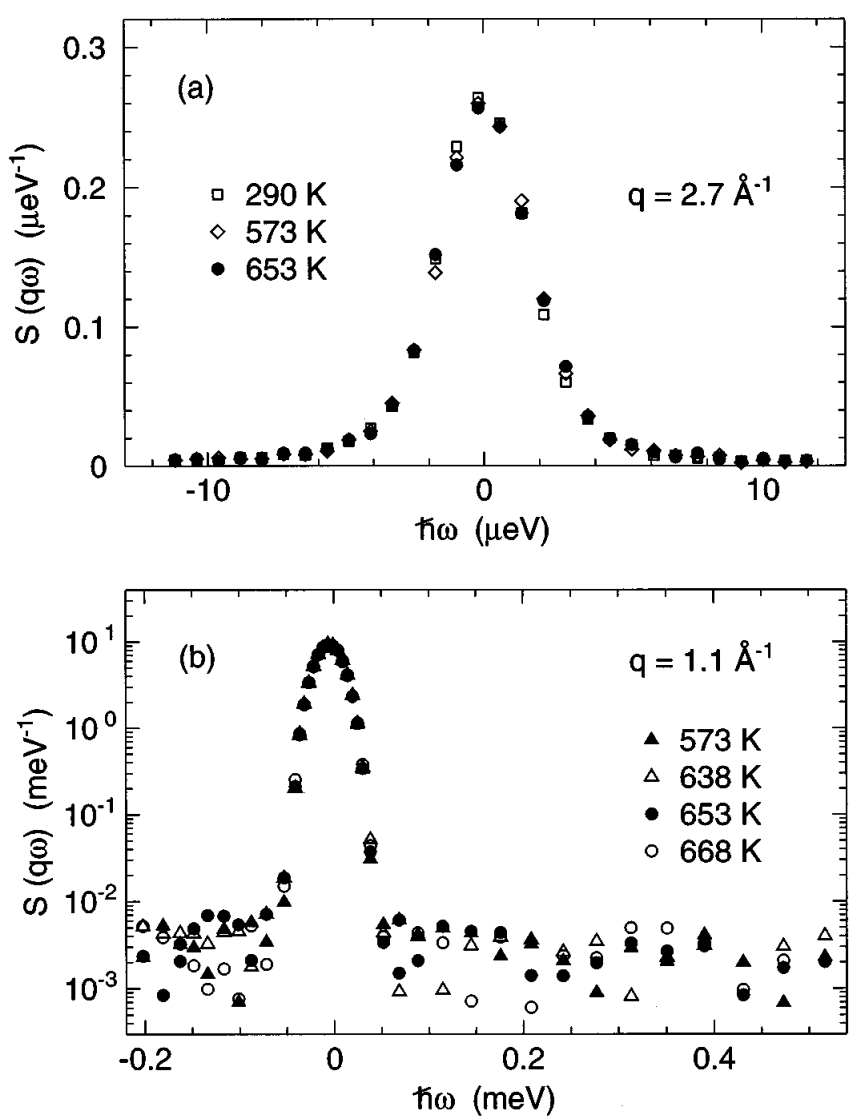

FIG. 5. Search for relaxational broadening in $S(q \omega)$ of ZrTiCuNiBe. (a) High-resolution spectra at IN10, rescaled to equal areas under the peak. At $653 \mathrm{~K}$ we could measure $7 \mathrm{~h}$ before the sample showed first signs of crystallization. No quasielastic broadening was observed. (b) Time-of-flight measurements at Mibémol. Within the noise level of $3 \times 10^{-4}$ no fast relaxation is observed. At $668 \mathrm{~K}$, we could measure $2.5 \mathrm{~h}$ before crystallization set in. Similar results were obtained for $\mathrm{ZrAlNiCu}$ up to $T=657 \mathrm{~K}$.

This negative result is fully consistent with recent measurements of viscosity ${ }^{16}$ and self-diffusion ${ }^{17}$ that revealed a rather moderate temperature dependence as compared to fragile systems. ${ }^{4}$ A crude estimate indicates that structural relaxation will fall into the window of neutron spectrometers only some $100 \mathrm{~K}$ above the highest temperature we could attain.

More promising is the search for a dynamic precursor of the glass transition, the fast $\beta$ relaxation, a localized rattling motion predicted by mode-coupling theory ${ }^{18}$ and found in neutron and light scattering spectra of molecular liquids on a psec scale. Recent molecular-dynamics simulations of a $\mathrm{Ni}_{50} \mathrm{Zr}_{50}$ melt strongly suggest that $\beta$ relaxation as a precursor of structural relaxation occurs also in metallic alloys. ${ }^{19}$ By neutron scattering the onset of anharmonic localized motion can be recognized even without measuring full spectra from a nonlinear decrease in elastic intensity. We have therefore performed two experiments: high-resolution spectral measurements on Mibémol, and elastic scans on IN10.

On the Mibémol spectrometer, an exceptionally good signal-to-noise ratio, better than 1000:1, could be achieved.

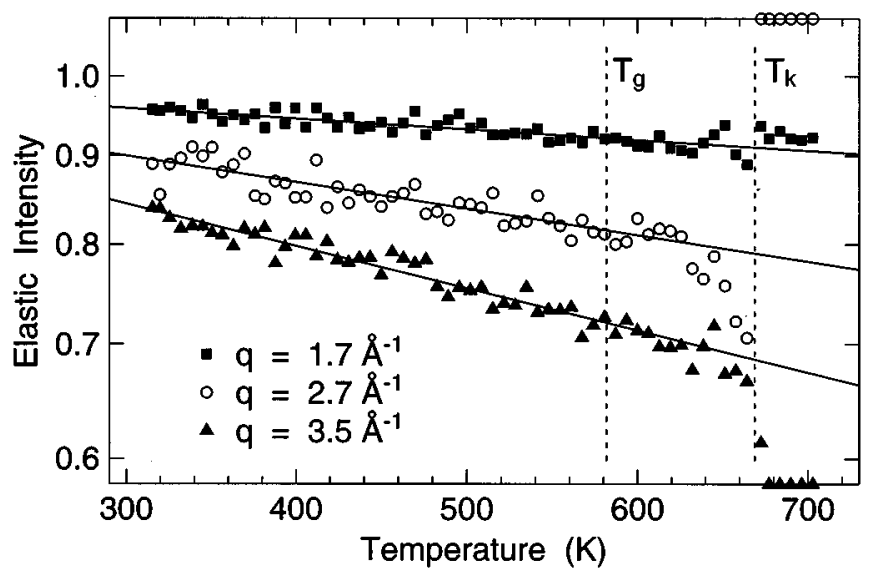

FIG. 6. Elastic scattering intensity $I(q, T)$ of $\mathrm{ZrTiCuNiBe}$ measured on IN10 with a constant heating rate of $0.2 \mathrm{~K} / \mathrm{min}$ and normalized to $I(q, T=0)=1$. Solid lines show the regular temperature dependence of a harmonic Debye-Waller factor; dashed vertical lines indicate the approximative temperatures of glass transition and crystallization. While the vibrational behavior does not change at $T_{g}$, we possibly observed the onset of relaxational motion at the structure factor maximum $q=2.7 \AA^{-1}$ in a $20 \mathrm{~K}$ interval before the sample crystallized at $T_{k}=670 \mathrm{~K}$.

Nevertheless, up to the highest temperatures [668(657) K for $\mathrm{ZrTiCuNiBe}(\mathrm{ZrAlNiCu})]$ elastic peaks coincide with the instrumental resolution, and there is no additional inelastic intensity [Fig. 5(b)]. The $\beta$-relaxation amplitude is expected to increase with $q$. The search for the onset of $\beta$ relaxation was therefore continued by measuring the elastic scattering intensity $I(q, T)$ on IN10 with its extended $q$ range. In Fig. 6, a perfectly exponential decay $I \propto \exp -q^{2}\left\langle u^{2}\right\rangle$ with $\left\langle u^{2}\right\rangle \propto T$ is found. This corroborates a main conclusion drawn from the inelastic spectra discussed before: our metallic glasses show even stronger harmonic behavior than most crystalline solids.

At the crystallization temperature $T_{k}$ the intensities at different detector angles suddenly increase or decrease as the static structure factor becomes sharply peaked. For $q=2.7 \AA^{-1}$ this change is preceded by an inflection of $I(q, T)$ in the opposite direction, beginning about $20 \mathrm{~K}$ below $T_{k}$. On the basis of the available data we can neither affirm nor exclude that this is an indication of fast liquid dynamics reaching the experimental window.

\section{CONCLUSION}

To summarize, the vibrational spectra of two amorphous metals show a pronounced low-frequency maximum that can be assigned to localized, strictly harmonic vibrations. Any physical interpretation of this "boson peak" should be able to cope with the fact that part of these modes are present also in the crystalline phase. With high-energy resolution spectroscopy, we have established a lower bound for structural relaxation falling into the dynamic range of neutron scatter- 
ing that is consistent with macroscopic measurements of transport coefficients. The occurrence of $\beta$ relaxation can be excluded up to at least $1.14 T_{g}$. This is in contrast to a fragile liquidlike $o$-terphenyl where the anharmonic decrease of $I(q T)$ begins even below $T_{g},{ }^{20}$ and where at $T_{c}=1.18 T_{g}$ the full critical dynamics of mode-coupling theory falls into the window of available neutron spectrometers. ${ }^{21}$ It appears that metallic melts are much less fragile than one would expect for a simple mixture of hard spheres; local structure and microscopic dynamics probably cannot be understood with- out proper consideration of the long-range and relatively strong metallic interaction. $^{22}$

\section{ACKNOWLEDGMENTS}

This work has been supported by the Deutsche Forchungsgemeinschaft (SPP Unterkühlte Metallschmelzen). We thank A. Magerl and G. Vogl for help in the development of new IN10 analyzers, G. André and C. Ritter for neutron-diffraction measurements, and W. Doster for a critical reading of the manuscript.
*Previously at INFP, Forschungszentrum Karlsruhe, 76021 Karlsruhe, Germany.

${ }^{1}$ Dynamics of Disordered Materials II, Proceedings Grenoble, edited by A. J. Dianoux, W. Petry, D. Richter [Physica A 201, 1 (1993)].

${ }^{2}$ A. P. Sokolov, A. Kisliuk, M. Soltwisch, and D. Quitmann, Phys. Rev. Lett. 69, 1540 (1992).

${ }^{3}$ L. Börjesson, A. K. Hassan, J. Swensson, and L. M. Torell, Phys. Rev. Lett. 70, 4027 (1993).

${ }^{4}$ R. Böhmer, K. L. Ngai, C. A. Angell, and D. J. Plazek, J. Chem. Phys. 99, 4201 (1993).

${ }^{5}$ T. Zhang, A. Inoue, and T. Masumoto, Mater. Trans. JIM 32, 10005 (1991).

${ }^{6}$ A. Peker and W. L. Johnson, Appl. Phys. Lett. 33, 2342 (1993).

${ }^{7}$ The $q$ range of IN10 was extended by a new $\mathrm{Si}_{311}$ monochromator and corresponding analyzer plates in a collaboration of TU München, Uni Wien and ILL Grenoble.

${ }^{8}$ M. M. Bredov et al., Sov. Phys. Solid State 9, 214 (1967).
${ }^{9}$ J. B. Suck et al., J. Phys. C 14, L167 (1980).

${ }^{10}$ J. B. Suck et al., J. Phys. C 14, 2305 (1981).

${ }^{11}$ J. B. Suck et al., Phys. Rev. Lett. 50, 49 (1983).

${ }^{12}$ J. Hafner, J. Phys. C 14, L287 (1981).

${ }^{13}$ U. Buchenau et al., Phys. Rev. Lett. 73, 2344 (1994).

${ }^{14}$ A. P. Sokolov et al., J. Noncryst. Solids 172-174, 138 (1994).

${ }^{15}$ W. Petry and J. Wuttke, Transp. Theory Stat. Phys. 24, 1075 (1995).

${ }^{16}$ R. Rambousky, M. Moske, and K. Samwer, Mater. Sci. Forum 179-181, 761 (1995).

${ }^{17}$ U. Geyer et al., Phys. Rev. Lett. 75, 2364 (1995).

${ }^{18}$ W. Götze and L. Sjögren, Transp. Theory Stat. Phys. 24, 801 (1995).

${ }^{19}$ H. Teichler, Phys. Rev. Lett. 76, 62 (1996).

${ }^{20}$ W. Petry et al., Z. Phys. B 83, 175 (1991).

${ }^{21}$ J. Wuttke et al., Z. Phys. B 91, 357 (1993).

${ }^{22}$ C. Hausleitner and J. Hafner, Phys. Rev. B 45, 115 (1992); 45, 128 (1992) 